\title{
Multiwavelength Study of Pulsation and Dust Production in Mira Variables Using Optical Interferometry for Constraints
}

\author{
M. J. Creech-Eakman ${ }^{1,2}$, J. Hora ${ }^{3}$, Z. Ivezic ${ }^{4}$, C. Jurgenson ${ }^{2}$, D. Luttermoser ${ }^{5}$, M.

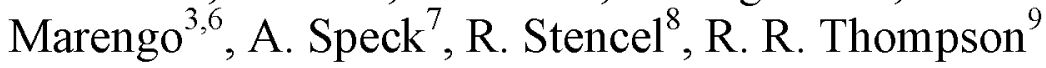 \\ 1 - New Mexico Institute of Mining and Technology, Department of Physics,801 Leroy Place, Socorro, NM, 87801 \\ 2 - Magdalena Ridge Observatory Interferometer, NMT,801 Leroy Place, Socorro, NM, 87801 \\ 3 - Harvard-Smithsonian Center for Astrophysics, 60 Garden St., MS 65, Cambridge, MA, 02138 \\ 4 - University of Washington, Department of Astronomy, Box 351580, Seattle, WA, 98195 \\ 5 - East Tennessee State University, Department of Physics, Box 70652, Johnson City, TN, 37614 \\ 6 - Iowa State University, Department of Physics and Astronomy, Ames, LA, 50011 \\ 7 - University of Missouri, Department of Physics and Astronomy, Columbia, MO, 65211 \\ 8 - University of Denver, Department of Physics and Astronomy, 2112 E. Wesley, Denver, CO, 80208 \\ 9 - Optical Sciences Corporation, SOFLA Project, NASADryden Aircraft Operations Facility, MS-S231, 2825 E. \\ Ave P, Bldg 703, Palmdale, CA, 93550
}

\begin{abstract}
Optical interferometry is a technique by which the diameters and indeed the direct pulsations of stars are routinely being measured. As a follow-on to a 7 year interferometric campaign to measure the pulsations of over 100 mira variables, our team has been using the Spitzer Space Telescope to obtain 95 mid-infrared spectra of 25 miras during their pulsations over one year while simultaneously ascertaining their near-infrared diameters using the Palomar Testbed Interferometer. These data will then be combined with modeling from NLTE and radiative transfer codes to place hard constraints on our understanding of these stars and their circumstellar environments. We present some initial results from this work and discuss the next steps toward fully characterizing the atmosphere, molecular photosphere and dust production in mira variables.
\end{abstract}

Keywords: mira variables, dust production, infrared spectroscopy, optical/infrared interferometry, facility: Spitzer Space Telescope facility: Palomar Testbed Interferometer

PACS: $95.85 . \mathrm{Gn}, 95.85 . \mathrm{Hp}, 95.85 . \mathrm{Jq}, 97.10 . \mathrm{Ex}, 97.10 . \mathrm{Me}$, 97.30.Jm

\section{INTRODUCTION}

In the past decade, astronomers have been able to create a much more complete picture of the extended atmosphere of mira variables. Much of this work is based on multi-wavelength studies of various components or regions in these atmospheres including masers and radio photospheres (Reid \& Menten, 1997 Cotton et al. 2008), molecular photospheres, especially with optical/infrared interferometry (Ragland et al. 2008, Perrin et al. 2004), and dust production, especially with respect to pulsational phase (Onaka et al. 2002, Matsuura et al. 2002, Marengo et al. 2007). This study seeks to increase our information on the interaction between pulsation and dust production by obtaining simultaneous data from space (infrared spectroscopy) and the ground (infrared interferometry) on a chemically complete sample of mira variables.

\section{MAIN COMPONENTS IN OUR STUDY}

This study relies on three key pieces of information: a) interferometric data to measure the diameter and derive a surface temperature of the molecular photosphere of the mira; b) synoptic sampling of the dust regions of the circumstellar environment using mid-infrared spectra; and c) complete modeling (hydrodynamics, physics, chemistry and radiative transfer) of the mira atmosphere and circumstellar region to develop a complete picture of the mira environment. Below we 
briefly describe what is available as part of this study in each of these areas.

\section{Palomar Testbed Interferometer Infrared Interferometric Data}

The Palomar Testbed Interferometer (PTI) was operated through the NASA Exoplanet Science Institute (NExScI) (formerly the Michelson Science Center) on the Caltech Campus from 1999-2009. It was a 3 element interferometer with baselines ranging from 85 to $110 \mathrm{~m}$ operating in the near-infrared mainly at $\mathrm{H}$ and $\mathrm{K}$ band, which yields spatial resolutions (for uniform disks) of 1.0 to about 5.5 milliarcseconds. All the data taken for this program were taken in the Kband filter, with low spectral dispersion of 0.1 microns per spectral channel in a queue scheduled mode.

Data were taken as nearly simultaneously with Spitzer as possible, only feasible due to the flexible queue scheduling at PTI. Reasons for not obtaining simultaneous data fell into three categories: poor sidereal position with respect to the Sun, cloudy weather, or miras too faint in the optical to acquire with the PTI star trackers (fainter than about $11^{\text {th }}$ magnitude at R). Calibrators were selected from our long-term PTI monitoring program on miras and typically were smaller than 0.8 milliarcseconds.
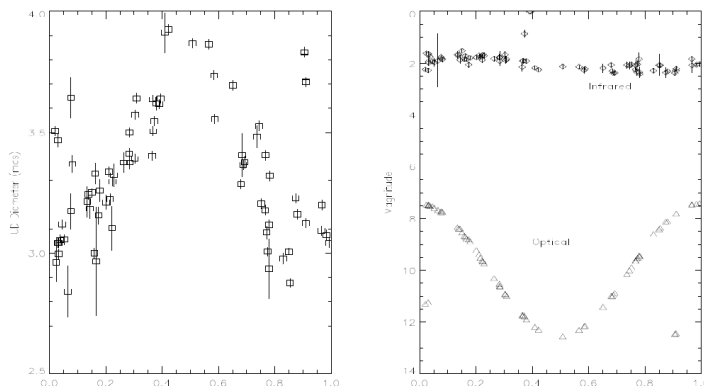

FIGURE 1. We present a sample of PTI data (L) and photometric levels (R) overlayed with AAVSO optical light curves, all modulo phase, for mira R Boo.

Typical PTI K-band visibilities on miras, which are very bright in the near-infrared, have error bars on the order of a few percent or less, translating to an uncertainty in the physical diameter of the mira which is dominated by the distance determination for the star (typically ranging from $5-20 \%$ uncertainties). We are also able to recover the NIR broad-band photometric levels using PTI data with approximately 10\% error bars. Typical PTI spectro-interferometric data show the basic spectral shape and angular size across Kband and allow one to distinguish molecular features such as $\mathrm{H}_{2} \mathrm{O}, \mathrm{CO}$ and $\mathrm{HCN}$ (Thompson et al., 2002). This, along with a distance determination and NIR photometric levels, allows us to make an unambiguous determination of the physical size, temperature and instantaneous bolometric flux of the molecular photosphere.

\section{Spitzer Space Telescope Synoptic Spectral Data}

The Spitzer Space Telescope data were all taken using the IRS instrument in order to obtain full wavelength coverage at the highest spectral resolution possible. Because the miras are very bright in the midIR, the SNR of the continuum can be several hundred, and can range into the thousands for bright emission lines. A typical full IRS spectrum took 12-15 minutes including pointing and backgrounds, which were taken for all stars in the dataset. Most stars were observed an average of 4 times over the period of approximately 10 months during Cycle 5.

The stars were chosen to span all the chemical subtypes, M, S and C, and taken from the PTI Mira Monitoring program, which included over 100 AGB stars. This allowed us to choose those sources which were of the correct diameter (approximately 2.5-3.5 mas) to get very high-quality measurements of the angular diameters. It also allowed us to choose stars that were in the "sweet spot" with regard to Spitzer's pointing, so that repeated visits could be scheduled with relative ease. We also knew, from previous PTI experience, that most of these miras could be optically tracked by PTI throughout their pulsation cycles, which was key to syncing the observations with Spitzer.

\section{Ancillary Data}

Ancillary data from the AAVSO archives and the Hipparcos database will also be used to constrain the measurements for input to the models. AAVSO data has excellent coverage for most miras, given their inherent magnitude range (up to 8 visual magnitudes) during their pulsation, giving us an extremely reliable measure of the pulsational phase of the mira. Further, for about $75 \%$ of the sample, Hipparcos measurements were able to be reliably reduced, producing distance measures accurate to $5-20 \%$, placing most miras in the $500 \mathrm{pc}$ to $2 \mathrm{kpc}$ distance range.

\section{Need for Complete Modeling of the Mira Atmosphere}

Anyone who studies miras is unlikely to disagree with the statement that their atmospheres and circumstellar environments are complicated to model. 
These complications are compounded by the number of unknowns typically associated with characterizing an atmosphere, including: physical location and temperature at the base of the atmosphere, pulsational cycle, shock location, bolometric flux, molecular species present, NLTE conditions and dust species undergoing formation and evolution in the outer layers of the atmosphere. With the combination of PTI, Spitzer, AAVSO and Hipparcos data, we are now poised to potentially build a realistic mira atmosphere.

Three key components must be used for this modeling effort. The first of these is the use of realistic hydrodynamical code to produce the correct input physics at the base of the atmosphere. These codes exist today in ongoing work begun by Hofner \& Dorfi (1992) and Bowen (1988), and now simply require some constraining information to allow one to select the proper model parameters for a particular realistic mira atmosphere. The second component is the calculation of NLTE departure coefficients and ionization fractions for all relevant atomic and molecular species. This will be accomplished within our team under the direction of Luttermoser using the radiative PANDORA code (c.f. Luttermoser, 1992). From here we use ATLAS to calculate a realistic stellar atmosphere which now has both realistic hydrodynamics and NLTE calculations as part of the physical calculations. The third step is to allow this atmosphere to impinge upon the dust forming region in the mira. From the spectra themselves we can identify the particular dust species present (c.f. Speck et al. 2006), and then use this to determine opacity coefficients to use with DUSTY radiative transfer code (Ivezic \& Elitzur, 1995) in order to model the molecular and chemical species present, and the effect that the impinging atmosphere has on the dust formation process.

The next steps, of course, require iterating on the above described process until we arrive at the correct set of assumptions that reproduces the measurements made with PTI, Spitzer and AAVSO.

\section{DATA COLLECTED FOR THE STUDY}

All PTI and Spitzer data for this project were collected between June, 2008 and March, 2009. In total PTI observations were executed on a monthly basis yielding over 60 individual visits to the 25 miras in the sample, each with roughly 4 scans per star and associated calibrator(s). With this we also have archival information (not simultaneous) of more than 15,000 individual visits of 100 miras (including these 25) over a 7-year time frame (Creech-Eakman \& Thompson, 2007) which can be used to fill in gaps in coverage when simultaneous measurements failed.
Spitzer observations numbered 95 full spectra (see Table 1) on all the targets in the sample, where the average number of visits per target was 4 in the 10 month period. This is over a factor of 8 times as many spectra of variable mira atmospheres as was able to be acquired during the ISO observing campaign, and thus represents a significant database for the astrophysical community. The phase coverage implied here is roughly $20 \%$ phase change for a typical 350 day mira period, which should yield adequate cyclic changes to thoroughly study the interplay between atmospheric physics and chemistry leading to the formation of dust. Unfortunately, at the present, these Spitzer data are somewhat non-trivial to reduce due to the brightness of the miras (compared to typical Spitzer targets). Significant work will have to be done to removing known fringing effects on the detector in order to derive realistic spectro-photometric levels.

TABLE 1. Spitzer data by chemical subclass and number of total scans. Unk = unknown chemical subtype and $\mathrm{NF}=$ no feature.

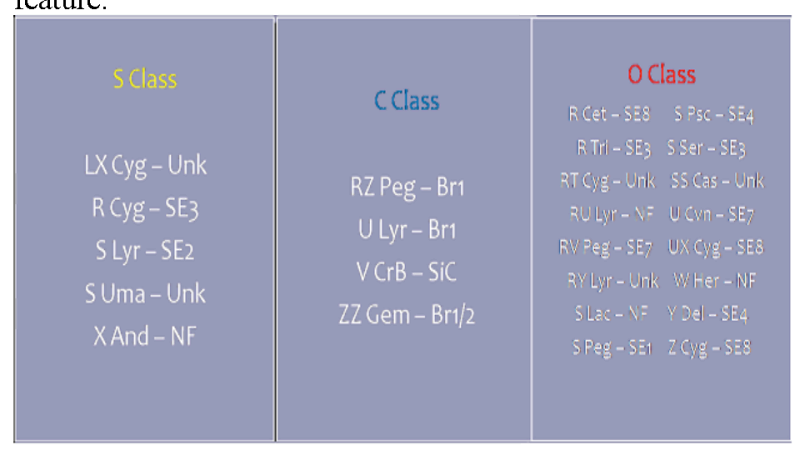

\section{MODELING PLANS}

Because the modeling is non-trivial, we have created a flow-diagram of the steps required to complete the process to arrive at a realistic explanation of the astrophysics present in each of these snapshots of variable atmospheres (Figure 2). We expect the process to take us several years to fully develop all tools and a consistent methodology. At the end, we hope to produce a website which will include the spectra themselves, the interferometric data, and the model outputs, so that as codes improve/develop and more realistic input physics models are derived, future groups can also use the data to constrain their own modeling efforts. 


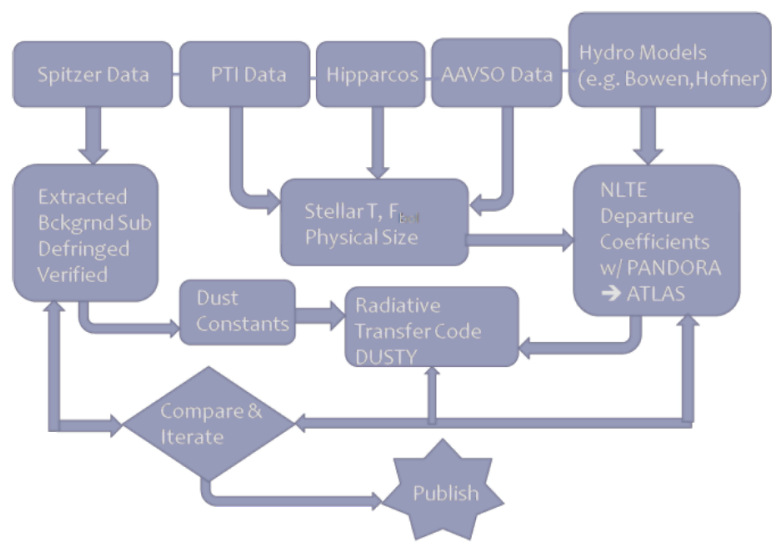

FIGURE 2. A complicated flow-chart diagram showing the interative components involved between data and modeling to produce realistic mira atmospheres.

Below we show, in Figure 3, one type of very simple output that can be expected using DUSTY, a generic silicate dust, and only the changes in angular diameter measured by PTI for the star R Boo. This is to say, there is no attempt here in this simulation to employ realistic atmospheric physics of the star using PANDORA, ATLAS or hydrodynamics and thus the atmosphere was modeled as a blackbody with a temperature change derived from the PTI data and a distance measurement. Nevertheless, there is significant evidence for changes in the circumstellar dust shell with phase.

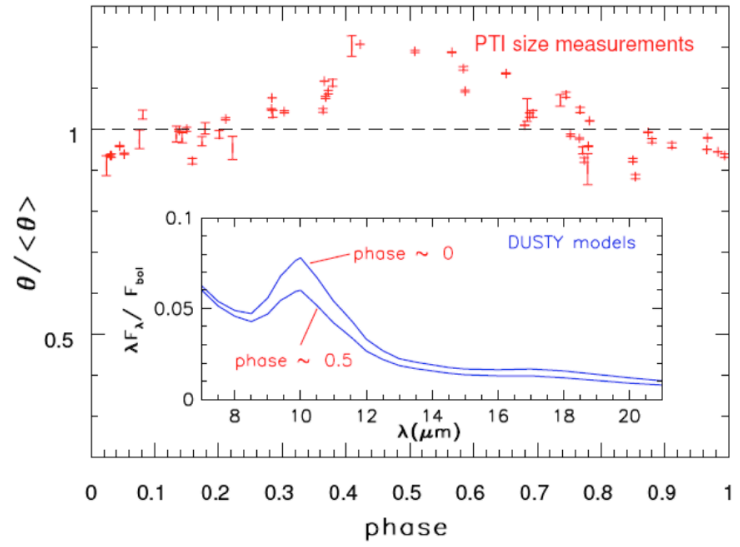

FIGURE 3. DUSTY model of R Boo circumstellar shell based on PTI input data, Hipparcos distance, and a generic silicate dust composition. See text for details.

\section{CONCLUSIONS}

It is our expectation that the PTI and Spitzer data, along with our modeling efforts, will yield a rich and exciting dataset that will keep many folks busy interpreting the circumstellar environs of miras for many years. This dataset will further demonstrate the power of using multi-wavelength, multiple technique studies to derive new information about stellar physics that was previously either too intractable, or perhaps considered "solved". We hope to publish much data from this project over the next several years and so encourage the reader to watch for our work in the future.

\section{ACKNOWLEDGMENTS}

The team wishes to thank the Spitzer Science Center and the IRS team for a wonderful spectrometer on the Spitzer telescope. We also thank the PTI collaboration and $\mathrm{K}$. Rykoski for many years of support of our Mira monitoring program. Finally, we acknowledge the use of SIMBAD, ADS Abstracts and AAVSO archives in the research and preparation of this manuscript. Some of the work on this project was supported through a NASA grant to the PI and team associated with Spitzer program GO50717.

\section{REFERENCES}

1. M. J. Reid and K. M. Menten. Astrophysical Journal, 476, 327 (1997).

2. W. D. Cotton, G. Perrin and D. Lopez, Astronomy and Astrophysics, 477, 853 (2008).

3. S. Ragland et al., Astrophysical Journal, 679, 746 (2008).

4. G. Perrin et al., Astronomy and Astrophysics, 426, 279 (2004).

5. T. Onaka, T. de Jong and I. Yamamura, Astronomy and Astrophysics, 388, 573 (2002).

6. M. Matsuura et al., Astronomy and Astrophysics, 383, 972 (2002).

7. M. Marengo et al. in "Why Galaxies Care about AGB Stars" ed. by Kerschbaum, Charbonnel and Wing, ASP Conference Series, 378 (2007).

8. R. R. Thompson, M. J. Creech-Eakman and G. T. van Belle, Astrophysical Journal, 577, 447 (2002).

9. S. Hofner and E. Dorfi, Astronomy and Astrophysics, 265, 207 (1992).

10. G. H. Bowen, Astrophysical Joumal, 329, 299 (1988).

11. D. G. Luttermoser, in "The Seventh Cool Stars Meeting", ed. by Giampapa and Bookbinder, ASP Conference Series, 26, 506 (1992).

12. A. K. Speck et al. Astrophysical Journal, 650, 829, (2006)

13. Z. Ivezic and M. Elitzur, Astrophysical Joumal, 445, 415 (1995).

14. M. J. Creech-Eakman and R. R. Thompson, in "The Biggest, Baddest, Coolest Stars", ed. by Luttermoser, Smith and Stencel, to be published by ASP Conference Series (2007) 